the inner and outer tubes, and further, the outer tube itself forms a most effective shield from external disturbances. The temperature of the concentric system was maintained constant by a steam jacket round the apparatus, while the temperatures of the oscillating crystal and the water vapour supply were stabilized by means of radiation thermostats, based on that developed in this Laboratory by Laby and Hopper ${ }^{2}$.

Results have been obtained for the refractive indexes of dry air and water vapour as follows: for dry air, at $58 \mathrm{Mc} . / \mathrm{sec} ., 100^{\circ} \mathrm{C} ., 76 \mathrm{~cm}$. mercury ;

$$
\mu=1 / 00024_{0} \pm 0 \cdot 000006 ;
$$

and for water vapour, at $58 \mathrm{Mc} / \mathrm{sec}, 100^{\circ} \mathrm{C} ., 76 \mathrm{~cm}$. mercury,

$$
\mu=\mathrm{I} \cdot 0030_{1} \pm 0 \cdot 00007 \text {. }
$$

It had been hoped to carry out work at other frequencies, temperatures and pressures, and with other gases, but this has been prevented by the War.

The important part played by the water vapour in the atmosphere in the propagation of ultra-short waves can be seen from these results. The figure for water vapour is found to agree with the value of 1.0060 for the dielectric constant of water vapour at $42 \mathrm{Mc} . / \mathrm{sec} ., 99 \cdot 8^{\circ} \mathrm{C}$. and $76 \mathrm{~cm}$. mercury, obtained by Tregigda ${ }^{1}$ using a heterodyne method.

The work, which was carried out under the direc. tion of Prof. T. H. Laby, was supported by the Australian Radio Research Board.

Natural Philosophy Department, F. J. KERR.

University of Melbourne. October 27.

1 Tregigda, Phys. Rev, , 57. 294-297 (1940).

Laby and Hopper, NATURE, 143, 240 (1939).

\section{Application of the Gibbs Adsorption Equation to Solutions of Paraffin-Chain Salts}

WITHYN recent years considerable doubt has been cast upon the validity of the Gibbs adsorption isotherm as applied to aqueous solutions of the paraf. fin-chain salts (soaps and soap-like molecules) ${ }^{1,2}$. The chief objection has been the numerous wellestablished examples of dilute solutions showing a minimum in the surface tension - concentration curve, usually at a surface tension of 30-35 dynes, presenting the paradox of a surface tension very much lower than that of water, and yet a zero or negative surface excess of solute as calculated from the Gibbs equation when applied in the customary manner.

Because of this apparent paradox, McBain and Mills $^{1}$, in a recent review, have concluded that the Gibbs equation is only a limiting law, and that terms allowing for the effects of orientation, of submerged double layer, and of free electrical charge (if any), should be included. This argument cannot be supported, however, since the systems under discussion are electrically neutral, and the other terms, assuming as they do a particular molecular interpretation of the surface layer, cannot be used as correcting factors, since they would be already included in the thermodynamically rigorous Gibbs equation.

Long and Nutting ${ }^{3}$ have recently put forward an explariation based upon the assumption that there is a surface layer of solute (giving positive adsorption at the interface) above a diffuse double layer of considerable depth, the concentrations in this double layer then determining whether the "Gibbsian surface excess" of solute is positive, zero or negative. (The hypothetical geometrical surface is chosen so that the surface excess of water is zero.) Unfortunately for this argument the total surface adsorp. tion as measured by the microtome method, and which would include both surface and diffuse layers, is always positive ${ }^{2}$. Other techniques support this invariable positive adsorption ${ }^{2}$.

The explanation suggested below, previously tentatively discussed by Powney and Addison ${ }^{4}$, would seem to provide a simple explanation of the above anomalies.

It is generally accepted that with the solutions under consideration micelle formation sets in at concentrations close to (probably rather less than) that at the minimum surface tension ${ }^{5}$. Also it seems well established that the micelle, owing to its structure, is precluded from existing in the surface layer, the surface active species being the single ionized molecule ${ }^{6}$. Thus the micelles may influence bulk properties such as freezing-point and conductivity, but can have little effect on the surface tension except in so far as their presence influences the concentration of single molecules in the solution. Hence the activity term in the Gibbs equation should be that for the molecularly dispersed solute in bulk solution ${ }^{7}$. The much larger amount of solute present in micelle form acts as a 'buffer', keeping the concentration of the molecularly dispersed solute sensibly constant, as shown by application of the Law of Mass Action, ${ }^{6,8}$. Accordingly, it is not surprising that, using the activity of the solution as a whole, as McBain and Mills, for example, have done ${ }^{1}$, the Gibbs equation should break down.

That the Gibbs equation holds, even with the paraffin-chain salts, when micelles are absent, is shown by the results of McBain and Wood with lauryl sulphonic acid ${ }^{2}$. Thus at a concentration of 0.002 gm.mol./l. (concentration at the minimum 0.006 gm.mol./l.), their measured adsorptions were $+2 \cdot 7$ and $+3 \cdot 0$ (microtome and interferometer respectively), that calculated by the Gibbs theorem being $+2 \cdot 4$ (gm.mol. $\left./ \mathrm{cm}^{2} \times 10^{10}\right)$.

The above explanation is further strengthened by calculation of the adsorption of lauryl sulphonic acid at the minimum surface tension ${ }^{9}$ which, assuming the adsorbed film to be monomolecular, gives a value of $+5 \cdot 7$, in good agreement with that using the microtome ${ }^{2}(+5 \cdot 4)$ (units as above). Further details will be published shortly.

Colloid Science Department,

$$
\text { A. E. Alexander. }
$$

The University,

Cambridge.

Nov. 18.

1 McBain and Mills, "Reports on Progress in Physics", 5, 30 (1989).

"McBain and Wood, Proc. Roy. Soc., A, 174, 286 (1940).

s Long and Nutting, J. Amer. Chem. Soc., 63, 625 (1941).

- Powney and Addison, Trans. Faraday Soc., 33, 1252 (1937).

${ }^{3}$ Lawrence, Annual Reports Chem. Soc., 37, 102 (1941).

- Murray, Trans. Faraday Soc., 31, 206 (1935); Alexander, Trans. Faraday Soc., 37, 15 (1941).

${ }^{7}$ cf. Nickerson, J. Phys. Chem., 40, 285 (1936).

- Bury and others, Phil. Mag., 4, 841 (1927); J. Chem. Soc., 679 (1929); Hartley, "Aqueous Solutions of Paraffin-Chain Salts" (Hermann, 1936).

Alexander, in the press. 